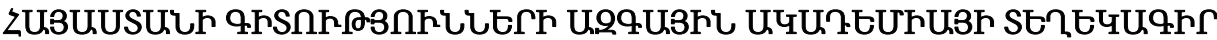 ИЗВЕСТИЯ НАЦИОНАЛЬНОЙ АКАДЕМИИ НАУК АРМЕНИИ
}

\author{
Uthuminlqu \\ 71, №3, 2018 \\ Механика \\ УДК 539.3
РАСПРОСТРАНЕНИЕ ВОЛН ТИПА SН В КОНЕЧНОПРОВОДЯЩЕМ
ВОЛНОВОДЕ \\ Геворкян А.В.
}

Ключевые слова: SH волна, проводящий, волновод

Gevorgyan A.V.

SH wave propagation in finite-conductive waveguide

Keywords: SH wave, conductive, waveguide

The SH wave propagation is considered in finite-conductive layer in outside constant magnetic field parallel to layer boundary. The dispersion equation is obtained. In long wave approximation the wave phase velocity is deduced and it is established that this velocity is more the waveguide material transverse velocity when magnetic field is absent.

\section{qunpqjui U.Ч.}

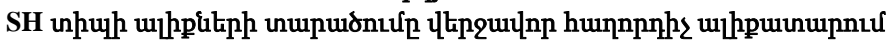

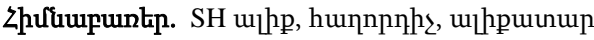

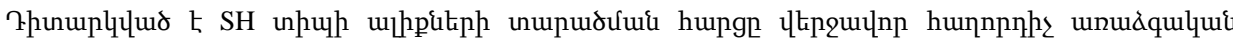

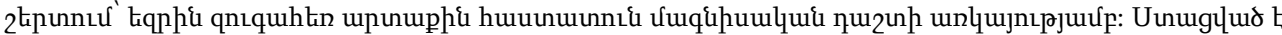

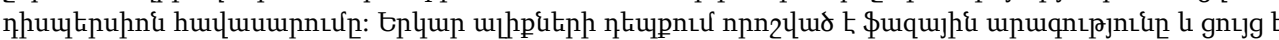

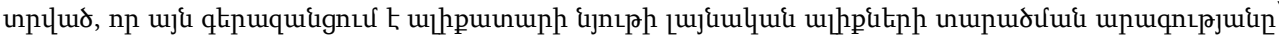

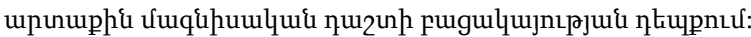

Рассматривается вопрос распространения SH волн в конечнопроводящем упругом слое при наличии внешнего постоянного магнитного поля, параллельного границе слоя. Получено дисперсионное уравнение.

В длинноволновом приближении определена фазовая скорость и установлено, что она превосходит скорость распространения поперечных волн материала волновода в отсутствие внешнего магнитнго поля.

Исследуется вопрос распространения волн типа SH в конечнопроводящем слое толщины $h$ при наличии внешнего постоянного магнитного поля.

Пусть упругий конечнопроводящий слой отнесён к прямугольной декартовой системе координат $O x_{1} x_{2} x_{3}$ : ось $x_{1}$ направлена вдоль границы слоя.

Начальное магнитное поле $\vec{H}_{0} \equiv\left[H_{0}, 0,0\right]$ направлено по оси $x_{1}$.

Магнитная проницаемость материала слоя принимается равной единице.

Учитывая, что в антиплоской деформации поле смещений и характеристики индуцированного электромагнитного поля в области $-h<x_{2}<0$ имеют вид

$\vec{u} \equiv\left[0,0, u_{3}\left(x_{1}, x_{2}, t\right)\right], \vec{h} \equiv\left[0,0, \mathrm{~h}_{3}\left(x_{1}, x_{2}, t\right)\right], \vec{e} \equiv\left[e_{1}\left(x_{1}, x_{2}, t\right), e_{2}\left(x_{1}, x_{2}, t\right)\right]$, из системы линеаризованных уравнений магнитоупругости, описывающей поведение электромагнитного поля и движение упругой проводящей среды, при пренебрежении таком смещения относительно тока проводимости получим следующую систему уравнений [1-3]: 
$c_{t}^{2} \nabla^{2} u_{3}+\frac{H_{0}}{4 \pi \rho} \partial_{1} h_{3}=\partial_{t}^{2} u_{3}$

$\nabla^{2} h_{3}-\frac{4 \pi \sigma}{c^{2}} \partial_{t} h_{3}=-\frac{4 \pi \sigma}{c^{2}} H_{0} \partial_{1} \partial_{t} u_{3}, \quad-h<x_{2}<0$

где $c_{t}^{2}=G / \rho$-квадрат скорости поперечных волн среды, $G$-модуль сдвига, $\rho$ плотность материала слоя, $\sigma$-удельная электропроводимость, $c$ электродинамическая постоянная.

Для вакуума в области $x_{2} \in(-\infty,-h) \cup(0, \infty)$ имеем уравнения Максвелла и вытекающие из них волновое уравнение

$\nabla \times \vec{h}^{*}=c^{-1} \partial_{t} \vec{e}^{*}, \quad \nabla \times \vec{e}^{*}=-c^{-1} \partial_{t} \vec{h}^{*}, \quad \nabla \cdot \vec{h}^{*}=0, \quad \nabla^{2} \vec{h}^{*}=c^{-2} \partial_{t}^{2} \vec{h}^{*}$

$\vec{h}^{*} \equiv\left[0,0, h_{3}^{*}\left(x_{1}, x_{2}, t\right)\right], \quad \vec{e}^{*} \equiv\left[e_{1}^{*}\left(x_{1}, x_{2}, t\right), \quad \vec{e}_{2}^{*}\left(x_{1}, x_{2}, t\right), 0\right]$.

Граничные условия рассматриваемой задачи имеют вид:

$\partial_{2} u_{3}=0, \quad h_{3}=h_{3}^{*}, \quad e_{1}=e_{1}^{*}, \quad x_{2}=0, \quad-h$

Решение системы уравнений (1) будем искать в виде

$\left(\begin{array}{l}u_{3} \\ h_{3}\end{array}\right)=\left(\begin{array}{l}A \\ B\end{array}\right) \cdot e^{k \lambda x_{2}} e^{i\left(k x_{1}-\omega t\right)}$

Подставляя (4) в (1), получим характеристическое уравнение системы (1) в виде

$\lambda^{4}-\left(2-\alpha z+z^{2}\right) \lambda^{2}+(1-\alpha z)\left(1+z^{2}\right)-\alpha s z=0$,

где

$\alpha=\frac{4 \pi \sigma c_{t}}{k c^{2}}, \quad z=\frac{i \omega}{k c_{t}}, \quad s=\frac{\mathrm{v}^{2}}{c_{t}^{2}}, \quad \mathrm{v}^{2}=\frac{H_{0}^{2}}{4 \pi \rho}$.

Корни уравнения (5) имеют следующий вид:

$\lambda_{[1,4]}= \pm \lambda_{ \pm}, \quad \operatorname{Re} \lambda_{ \pm}>0, \quad \operatorname{Re} z>0$

$\lambda_{ \pm}=\left\{\frac{2-\alpha z+z^{2}}{2} \pm\left[\left(\frac{2-\alpha z+z^{2}}{2}\right)^{2}-(1-\alpha z)\left(1+z^{2}\right)+\alpha s z\right]^{1 / 2}\right\}^{1 / 2}=$

$=\left\{\frac{2-\alpha z+z^{2}}{2} \pm\left[\left(\frac{z^{2}+\alpha z}{2}\right)^{2}+\alpha s z\right]^{1 / 2}\right\}^{1 / 2}$

Таким образом, решения системы (1) и волнового уравнения (2) запишутся в следующем виде:

$u_{3}=\left[A_{+}^{+} \cdot e^{k \lambda+x_{2}}+A_{+}^{-} \cdot e^{-k \lambda+x_{2}}+A_{-}^{+} \cdot e^{k \lambda-x_{2}}+A_{-}^{-} \cdot e^{-k \lambda-x_{2}}\right] e^{i\left(k x_{1}-\omega t\right)}$
$h_{3}=\left[B_{+}^{+} \cdot e^{k \lambda+x_{2}}+B_{+}^{-} \cdot e^{-k \lambda+x_{2}}+B_{-}^{+} \cdot e^{k \lambda-x_{2}}+B_{-}^{-} \cdot e^{-k \lambda-x_{2}}\right] e^{i\left(k x_{1}-\omega t\right)}$ 
$B_{ \pm}^{ \pm}=\frac{i k \alpha z H_{0}}{\lambda_{ \pm}^{2}+\alpha z-1} A_{ \pm}^{ \pm}, \quad B(\lambda)=\frac{i k \alpha z H_{0}}{\lambda^{2}+\alpha z-1} A(\lambda)$

$h_{3}^{*}=\left[\begin{array}{cc}D_{-} \cdot e^{k v_{*} x_{2}} e^{i\left(k x_{1}-\omega t\right)}, & x_{2} \in(-\infty,-h) \\ D_{+} \cdot e^{k v_{*} x_{2}} e^{i\left(k x_{1}-\omega t\right)}, & x_{2} \in(0, \infty)\end{array}\right.$

$v_{*}=\sqrt{1+c_{t}^{2} \cdot c^{-2} z^{2}} \sim 1$

Подставляя выражения (7)-(9) в граничные условия (3), получим систему алгебраических однородных линейных уравнений для произвольных постоянных и из условия совместности этой системы получим дисперсионное уравнение в следующем виде:

$\left(\frac{\beta_{+} \lambda_{+}-\beta_{-} \lambda_{-}}{\beta_{+} \lambda_{+}+\beta_{-} \lambda_{-}}\right)^{2} \equiv \operatorname{th}^{2} \ln \sqrt{\frac{\beta_{+} \lambda_{+}}{\beta_{-} \lambda_{-}}}=\frac{\operatorname{sh}^{2}\left(k h \cdot \frac{\lambda_{+}-\lambda_{-}}{2}\right)}{\operatorname{sh}^{2}\left(k h \cdot \frac{\lambda_{+}+\lambda_{-}}{2}\right)}$

$\beta_{ \pm}=\lambda_{ \pm}^{2}+\alpha z-1$

Далее, в длинноволновом приближении, то есть $k^{2} h^{2} \ll 1$, заменяя гиперболические синусы своими аргументами, уравнение (10) переходит в совокупности уравнений

$\left[\begin{array}{l}\lambda_{+} \lambda_{-}=0 \\ \left(\lambda_{+}^{2}-\lambda_{-}^{2}\right)^{2}=0 \\ 1+z^{2}=0\end{array} \Rightarrow\left[\begin{array}{l}\alpha z^{3}-z^{2}+\alpha(1+s) z-1=0 \\ z^{3}+2 \alpha z^{2}+\alpha^{2} z+4 \alpha s=0 \\ 1+z^{2}=0\end{array}\right.\right.$

Рассмотрим первое уравнение совокупности (11)

$z^{3}-\alpha^{-1} z^{2}+(1+s) z-\alpha^{-1}=0$.

Для хороших проводящих сред можно принять $\alpha^{2} \gg 1$ и в силу ограниченности значения напряжённости внешнего магнитного поля, реализуемого на практике постоянными магнитами, примем $s<0,1$.

Алгебраическое уравнение (12) имеет одно действительное отрицательное решение, не соответствующее условиям задачи, и комплексно сопряжённое решение с положительной действительной частью

$z_{1}=\sqrt[3]{-\frac{q}{2}+\sqrt{Q}}+\sqrt[3]{-\frac{q}{2}-\sqrt{Q}}+\frac{\alpha^{-1}}{3}>0$

$z_{ \pm}=\frac{\alpha^{-1}}{3}-\frac{\sqrt[3]{-\frac{q}{2}+\sqrt{Q}}+\sqrt[3]{-\frac{q}{2}-\sqrt{Q}}}{2} \pm i \sqrt{3} \cdot \frac{\sqrt[3]{-\frac{q}{2}+\sqrt{Q}}-\sqrt[3]{-\frac{q}{2}-\sqrt{Q}}}{2}$,

где 


$$
\begin{aligned}
& \frac{q}{2}=-\left(\frac{\alpha^{-1}}{3}\right)^{3}-\left(1-\frac{s}{2}\right) \frac{\alpha^{-1}}{3}<0, \quad \frac{P}{3}=\frac{1+s}{3}-\left(\frac{\alpha^{-1}}{3}\right)^{2}>0 \\
& Q=\left(\frac{q}{2}\right)^{2}+\left(\frac{P}{3}\right)^{3}=3\left(\frac{\alpha^{-1}}{3}\right)^{4}+\frac{1}{3}\left(2-5 s-\frac{s^{2}}{4}\right)\left(\frac{\alpha^{-1}}{3}\right)^{2}+\left(\frac{1+s}{3}\right)^{3}>0
\end{aligned}
$$

Предполагая, что искомая волна распространяется по положительному направлению оси $x_{1}$, в формуле (13) берётся знак плюс.

Так как

$$
\frac{i \omega}{k c_{t}}=z_{+}=\operatorname{Re} z_{+}+i \operatorname{Im} z_{+}
$$

то с учётом (7) определим фазовую скорость распространения SH волн в волноводе в направлении оси $x_{1}$

$\mathrm{v}_{\phi}=\operatorname{Im} z_{+} \cdot c_{t}=\frac{\sqrt{3}}{2}\left(\sqrt[3]{-\frac{q}{2}+\sqrt{Q}}-\sqrt[3]{-\frac{q}{2}-\sqrt{Q}}\right) c_{t}>c_{t}$

Теперь докажем, что $\operatorname{Im} z_{+}>1$, то есть,

$\sqrt[3]{-\frac{q}{2}+\sqrt{Q}}-\sqrt[3]{-\frac{q}{2}-\sqrt{Q}}>\frac{2}{\sqrt{3}}$

Возводя обе части неравенства в куб, получим:

$2 \sqrt{Q}+P\left(\sqrt[3]{-\frac{q}{2}+\sqrt{Q}}-\sqrt[3]{-\frac{q}{2}-\sqrt{Q}}\right)>\frac{8}{3 \sqrt{3}}$

C учётом (15) докажем

$2 \sqrt{Q}+\frac{2}{\sqrt{3}} P>\frac{8}{3 \sqrt{3}}$ или $\sqrt{Q}>\frac{1}{\sqrt{3}}\left(\frac{4}{3}-P\right)$.

При $0<P<\frac{4}{3}$, возводя обе части неравенства (17) в квадрат, после некоторых преобразований приходим к неравенству $\left(1-\frac{s}{4}\right) \alpha^{-2}+(s-3)^{2}>0, \quad s<0,1$.

Следовательно, справедливо неравенство (15), так как

$$
\left(\sqrt[3]{-\frac{q}{2}+\sqrt{Q}}-\sqrt[3]{-\frac{q}{2}-\sqrt{Q}}\right)^{3}>2 \sqrt{Q}+\frac{2}{\sqrt{3}} \mathrm{P}>\left(\frac{2}{\sqrt{3}}\right)^{3} .
$$

В последующей работе мы убедимся, что уравнение (12) представляет собой дисперсионное уравнение распространения SH волн в упругой конечнопроводящей 
среде, когда волновой вектор и вектор напряжённости внешнего магнитного поля равнонаправлены.

Второе уравнение совокупности (11)

$z^{3}+2 \alpha z^{2}+\alpha^{2} z+4 \alpha s=0$

при $s<0,1$ и $\alpha^{2} \gg 1$ имеет три вещественные отрицательные решения, не соответствующие условиям задачи:

$\frac{q}{2}=\alpha\left(2 s-\frac{\alpha^{2}}{27}\right), \quad \frac{P}{3}=-\left(\frac{\alpha}{3}\right)^{2}<0, \quad Q=4 s \alpha^{2}\left(s-\frac{\alpha^{2}}{27}\right)<0$.

Последнее уравнение совокупности (15) является частным случаем дисперсионного уравнения, соответствующего распространению SH волн при отсутствии внешнего магнитного поля, то есть при $s=0$, из (6) и (10) имеем:

$\lambda_{+}=\sqrt{1+z^{2}}, \quad \lambda_{-}=1, \quad \beta_{-}=0$

$\operatorname{ch}\left(k h\left(\lambda_{+}+\lambda_{-}\right)\right)-\operatorname{ch}\left(k h\left(\lambda_{+}-\lambda_{-}\right)\right)=2 \operatorname{sh}\left(k h \lambda_{+}\right) \cdot \operatorname{sh}\left(k h \lambda_{-}\right)=0$

$\operatorname{sh}\left(k h \sqrt{1-\frac{\omega^{2}}{k^{2} c_{t}^{2}}}\right)=i \cdot \sin \left(k h \sqrt{\frac{\omega^{2}}{k c_{t}^{2}}-1}\right)=0$.

В заключении отметим, что при $k h \rightarrow \infty$ уравнение (10) переходит в дисперсионное уравнение, соответствующее распространению поверхностных $\mathrm{SH}$ волн вдоль границы конечнопроводящего полупространства [4,5].

\section{ЛИТЕРАТУРА}

1. Новацкий В. Теория упругости. М.: Мир, 1975.

2. Седов Л.И. Механика сплошной среды. Т.1. М.: Наука, 1983.

3. Тамм И.Е. Основы теории электричества. М.: Наука, 1976.

4. Геворкян А.В. Магнитоупругие поверхностные сдвиговые волны в конечнопроводящем полупространстве. //Изв. НАН Армении. Механика. 1994. Т.47. №1-2. C.44-52.

5. Белубекян М.В., Геворкян А.В. Магнитоупругие поверхностные сдвиговые волны в конечнопроводящей среде. //Докл.НАН Армении. 1995. Т.95. №2. С.86-88.

\section{Сведения об авторе:}

Геворкян Артак Вараздатович - к.ф.-м.н., старший научный сотрудник Института механики НАН Армении.

Адрес: 0019, Ереван, пр. Маршала Баграмяна, 24/2,

Тел.: (+37477) 028889.

E-mail: AGevorgyanV@ gmail.com

Поступила в редакцию 20. 12. 2018 